\title{
Rational design of graphene oxide and its hollow CoO composite for superior oxygen reduction reaction
}

\author{
Guoxin Zhang, Yuqi Xu, Lin Wang, Jindi Wang, Yun Kuang and Xiaoming Sun*
}

\begin{abstract}
Graphene oxide (GO) is an important derivative of graphene, fascinating the entire world with its dazzling properties and versatile performance. However, the synthesis of GO via chemical routes often results in limited control of the density of functionalities and their distribution, presenting a barrier to the spread of GO applications. We modified Hummers' method and aimed at controlling the oxygen functionality of GO. The highest oxygen content of the modified synthetic GO (MdGO) occurs at edge regions, and the large proportion of carboxyl groups can be easily removed upon annealing. The excellent conductivity of intrinsic graphene can thus be recovered after the removal of the main functional groups. The resulting MdGO was reduced and doped with $\mathrm{NH}_{3}$, and the reduced MdGO (rMdGO) was determined to be an excellent support for oxygen reduction reaction (ORR) electrocatalysts. As a demonstration, a composite of $\mathrm{CoO}$ and N-rMdGO was fabricated, which exhibited highly comparable ORR performance in alkaline relative to $20 \mathrm{wt} . \%$ $\mathrm{Pt} / \mathrm{C}$.
\end{abstract}

\section{INTRODUCTION}

The oxidized derivatives of graphene are intriguing 2D materials that are highly promising for various applications such as energy storage and conversion as well as supporting catalysts because of their unusual electrical and mechanical properties [1-6]. Their frameworks are widely open for functionalization, and extensive reports have indicated that the density and distribution of functional groups on the edge and basal plane of graphene oxide (GO) could hold the key to many properties and downstream applications [7-10]. High functionality, on one hand, engages good solubility for high processibility; on the other, it creates a large proportion of intrinsic defects that may greatly degrade the conductivity [11]. Although their atomically thin layer can provide sufficiently high surface area for catalyst loading, the $2 \mathrm{D}$ conductivity for excellent electron transportation should not be sacrificed, especially in the area of electrochemistry.

To balance the functionality and processibility of GO, great efforts have been devoted to the exploration of GO synthesis. Roughly, two strategies are involved; one is to mildly oxidize graphene with less addition of strong oxidizing agents, which leads to a relatively low yield of singleand few-layered GO $[4,5]$. The other secures a high yield of single-layered GO by applying strong oxidizing conditions but also induces irreparable damage to the conductivity of GO even with subsequent reduction [12-14]. Previous studies have indicated that epoxy and hydroxyl groups are widely accepted as the main functionalities of GO. With a harsh oxidization degree, carboxyl groups would be introduced [15]. The epoxy and hydroxyl groups are mainly distributed on the basal plane, while carboxyl groups are distributed on the edges. Most reduction methods are effective in the removal of these functional groups but lack power in defect repair $[12,14]$. Dai's group developed ball-milling fabrication of edge-functionalized few-layered graphene, the functional groups of which mainly consist of carboxyl groups on edge regions because of the reaction space limit during manufacturing [16]. The graphene of such a structure exhibits good solubility and conductivity, demonstrating high potential in electrochemical fields [16]. Therefore, aiming at electrochemical applications, the basal plane should be strictly controlled for the loading of functional groups to maintain the excellent conductivity of GO. However, currently, this method still challenges solution-based chemical routes.

In this study, we synthesized GO with most of its functional groups located at edge regions to control its defect density on the basal plane and provide good solubility. The resulting GO was thoroughly characterized to substantiate the forms and distribution of the functional groups. Unlike GO obtained using other synthetic methods, our modified synthetic GO (MdGO) was observed to overwhelmingly possess carboxyl groups rather than hydroxyl groups in regular Hummers' GO [15]. These carboxyl groups are capable of suspending GO in water and can be easily re-

State Key Laboratory of Chemical Resource Engineering, Beijing University of Chemical Technology, Beijing 100029, China.

* Corresponding author (email: sunxm@mail.buct.edu.cn) 
moved by annealing, especially for most oxygen functionalities distributed in the edge region. Sparsely distributed oxygen functionalities on the basal plane provide anchors for loading catalysts. In this study, $\mathrm{CoO}$ was used to form a composite with $\mathrm{NH}_{3}$-reduced MdGO (N-rMdGO). The composite was observed to be highly comparable to 20 wt.\% Pt/C in electrochemically catalyzing the oxygen reduction reaction (ORR), confirming the effective synthesis of the catalyst support of MdGO.

\section{EXPERIMENTAL SECTION}

\section{Materials}

Graphite powder (99.95\% purity, Aladdin), $\mathrm{H}_{2} \mathrm{O}_{2}$ ( 30 wt.\%), and $\mathrm{Co}(\mathrm{OAc})_{2} \cdot \mathrm{H}_{2} \mathrm{O}$ were purchased from XiLong Chemical Co., Ltd. The other materials, $\mathrm{KMnO}_{4}$, thioactamide, $\mathrm{H}_{2} \mathrm{SO}_{4}$ (98 wt.\%), $\mathrm{NH}_{3} \cdot \mathrm{H}_{2} \mathrm{O}$, condensed $\mathrm{HCl}$, and dimethyl sulfoxide (DMSO) were all of AR grade, purchased from Beijing Chemical Reagent Co., Ltd, and used as received.

\section{Modified synthesis of graphene oxide (MdGO)}

In a typical synthesis procedure, $3.0 \mathrm{~g}$ graphite powder was first added into $108.0 \mathrm{~mL}$ of condensed $\mathrm{H}_{2} \mathrm{SO}_{4}$ (98 wt.\%), followed by the addition of $3.0 \mathrm{~g} \mathrm{KMnO}_{4}$. Then, the container was sealed with Biopute parafilm to prevent the contact of $\mathrm{H}_{2} \mathrm{SO}_{4}$ with moisture in the air. The suspension was mechanically stirred for three days to fully engage $\mathrm{H}_{2} \mathrm{SO}_{4}$ intercalation. Three additions of $10.0 \mathrm{~mL}$ of deionized water at an interval of $5 \mathrm{~min}$ were performed to increase the temperature to $\sim 70^{\circ} \mathrm{C}$. The oxidation began once water was introduced. After a short period, the oxidation was terminated with the addition of $100.0 \mathrm{~mL}$ of water because $\mathrm{H}_{2} \mathrm{SO}_{4}$ of any concentration lower than $70 \mathrm{wt}$.\% will lose its oxidative capability [17]. The suspension was continuously stirred until the temperature reached lower than $30^{\circ} \mathrm{C}$; then, $10.0 \mathrm{~mL}$ of $\mathrm{H}_{2} \mathrm{O}_{2}$ (30 wt.\%) was introduced. Centrifugation and washing were then performed to obtain clean MdGO. Hummers' GO (HGO) was prepared following a previously reported route [18].

\section{Reduction and N-modification of MdGO and HGO}

Hydrothermal treatment is effective for incorporating molecules into defects of GO. We used a hydrothermal method to reduce and functionalize $\mathrm{MdGO}$ and $\mathrm{HGO}$ at $120^{\circ} \mathrm{C}$. The introduction of $\mathrm{N}$-dopant was achieved using $\mathrm{NH}_{3} \cdot \mathrm{H}_{2} \mathrm{O}[12,13]$. Afterwards, the hydrothermally treated product was dissolved in DMSO to attain a concentration of $1.0 \mathrm{mg} \mathrm{mL}^{-1}$ and was thoroughly sonicated before use.

\section{Preparation of $\mathrm{CoO} / \mathrm{N}-\mathrm{rMdGO}$ composite}

A facile wet dip method was applied for the preparation of the $\mathrm{CoO} / \mathrm{N}-\mathrm{rMdGO}$ and $\mathrm{CoO} / \mathrm{N}-\mathrm{rHGO}$ hybrids. The typical procedure was as follows: $1.0 \mathrm{mmol} \mathrm{Co}(\mathrm{OAc})_{2}$ was dissolved into $10.0 \mathrm{~mL}$ of a DMSO suspension of N-rMdGO $\left(\sim 1 \mathrm{mg} \mathrm{mL} \mathrm{mL}^{-1}\right)$ and sonicated for $30 \mathrm{~min}(23 \mathrm{kHz}, 240$ $\mathrm{W})$. Then, the suspension was magnetically stirred for 2 $\mathrm{h}$ at $80^{\circ} \mathrm{C}$. Centrifugation was then used to obtain a solid product, which was dried in a $60^{\circ} \mathrm{C}$ oven. Further annealing under $\mathrm{N}_{2}$ ambient at $500^{\circ} \mathrm{C}$ resulted in crystallized CoO on N-rMdGO.

\section{Characterization}

The morphologies and structures of the materials were examined using scanning electron microscope (SEM, Zeiss SUPRA55), transmission electron microscope (TEM, Hitachi 800), high-resolution scanning electron microscope (HRTEM, JEOL JEM-2100), and X-ray photoelectron spectroscope (XPS, Thermo Electron ESCALAB 250). Powder X-ray diffraction (XRD) patterns of the samples were recorded on a Shimadzu XRD-6000. Raman spectra were recorded on a LabRAM Aramis Raman spectrometer (HORIBA Jobin Yvon).

\section{Electrochemical measurements}

For these measurements, $5.0 \mathrm{mg}$ catalyst and $50 \mu \mathrm{L}$ of $\mathrm{Na}$ fion (DuPont, $5 \mathrm{wt} . \%$ ) were dispersed in $1.0 \mathrm{~mL}$ of $\mathrm{N}, \mathrm{N}$-dimethylformamide (DMF) with at least 10-min sonication to form a homogeneous ink. Then, $5 \mu \mathrm{L}$ of ink (containing $25 \mu \mathrm{g}$ catalyst) was loaded on a 5.0 -mm-diameter glassy carbon rotating disk electrode (Pine Instruments), yielding a loading of $0.1274 \mathrm{mg} \mathrm{cm}^{-2}$. Cyclic voltammetry (using a Princeton Applied Research PARSTAT 2273 potentiostat) was conducted in an electrochemical cell using a saturated calomel electrode (SCE, 0.99 vs. reversible hydrogen electrode in $0.1 \mathrm{~mol} \mathrm{~L}^{-1} \mathrm{KOH}$ ) as the reference electrode, a 1.0 $\mathrm{cm}^{2}$ platinum foil as the counter electrode, and the sample modified glassy carbon electrode as the working electrode. The electrolyte $\left(0.1 \mathrm{~mol} \mathrm{~L}^{-1} \mathrm{KOH}\right)$ was saturated with oxygen by bubbling $\mathrm{O}_{2}$ before the measurement. The flow of $\mathrm{O}_{2}$ was maintained over the electrolyte during the recording of cyclic voltammograms (CVs). The working electrode was cycled at least 5 times before data were recorded at a scan rate of $50 \mathrm{mV} \mathrm{s}^{-1}$. For the rotating disk electrode (RDE) measurements, the working electrodes were scanned cathodically at a rate of $5 \mathrm{mV} \mathrm{s}^{-1}$ with varying rotating speeds from 400 to $2025 \mathrm{rpm}$. Koutecky-Levich (K-L) plots $\left(J^{-1} v s\right.$. $\left.\omega^{-1 / 2}\right)$ were analyzed at various electrode potentials. The slopes of the linear fit lines were used to calculate the number of electrons transferred $(n)$ based on the K-L equation: 


$$
\begin{gathered}
\frac{1}{J}=\frac{1}{J_{\mathrm{L}}}+\frac{1}{J_{\mathrm{K}}}=\frac{1}{B \omega^{1 / 2}}+\frac{1}{J_{\mathrm{K}}}, \\
B=0.62 n F C_{\mathrm{o}}\left(D_{\mathrm{o}}\right)^{2 / 3} v^{-1 / 6}, \\
J_{\mathrm{K}}=n F k C_{o},
\end{gathered}
$$

where $J$ is the measured current density, $J_{\mathrm{K}}$ and $J_{\mathrm{L}}$ are the kinetic- and diffusion-limiting current densities, $\omega$ is the angular velocity, $n$ is the transferred electron number, $F$ is the Faraday constant, $C_{0}$ is the bulk concentration of $\mathrm{O}_{2}, D_{0}$ is the $\mathrm{O}_{2}$ diffusion coefficient, $v$ is the kinematic viscosity of the electrolyte, and $k$ is the electron-transfer rate constant. For the Tafel plot, the kinetic current was calculated from the mass-transport correction of RDE using

$$
J_{\mathrm{K}}=\frac{J \times J_{\mathrm{L}}}{\left(J_{\mathrm{L}}-J\right)} .
$$

\section{RESULTS AND DISCUSSION}

The strategy follows the commonly used Hummers' method with a few adjustments, i.e., much longer intercalation and shorter reaction duration [19]. The oxidation of graphite was performed using regular oxidizing agents, condensed $\mathrm{H}_{2} \mathrm{SO}_{4}$ and $\mathrm{KMnO}_{4}$, and the mixture was stirred continuously for three days at low temperature $\left(<20^{\circ} \mathrm{C}\right)$ to fully engage the intercalation and low enough oxidation of graphite. Subsequently, a medium volume of water was added to the mixture to elevate the reaction temperature to a desirable level to control the reaction at a certain intensity. An excess water addition will soon increase the temperature to above $90^{\circ} \mathrm{C}$, which will introduce a high oxygen content on the basal plane, while deficient water will result in too low a yield of single- and few-layered GO.

The MdGO was first characterized by XPS to reveal the details of the functional groups and the results were compared with those of Hummers' GO (HGO). The XPS C1s spectrum of MdGO and its deconvoluted curves for specific functional groups are presented in Fig. 1a and compared with the typical C1s spectrum of HGO. MdGO exhibited a remarkably high content of carboxyl groups, much higher than that of hydroxyl groups. We calculated the percentage of these two types of functional groups in each material and observed that the ratio of $\mathrm{C}=\mathrm{O} / \mathrm{C}-\mathrm{O}$ in $\mathrm{MdGO}$ was roughly 2, whereas that in $\mathrm{HGO}$ was 0.17 , as observed in Fig. 1e. This distinct difference can also be observed in the $\mathrm{O} 1 \mathrm{~s}$ spectrum in Figs. $1 \mathrm{c}$ and d. A similar tendency of the $\mathrm{O}$ configurations could be identified. The figure of $\mathrm{C}=\mathrm{O} / \mathrm{C}-\mathrm{O}$ reaches $\sim 5$ because of the high number of surface-attached
$\mathrm{CO}_{2}$ molecules, as demonstrated in Figs. $1 \mathrm{c}$ and d. $\mathrm{CO}_{2}$ is a common adsorbed molecule that is inevitably present on the surface of nanomaterials [20,21]. The Raman characterization revealed that $\mathrm{MdGO}$ had a much lower defect level $\left(I_{\mathrm{D}} / I_{\mathrm{G}} \approx 0.86\right)$ than $\mathrm{HGO}\left(I_{\mathrm{D}} / I_{\mathrm{G}} \approx 1.14\right)$ (Fig. 1f), which should be related to the abnormal distribution of functional groups. Abundant polar functional groups can be easily accessed and solvated by water molecules. Thus, MdGO exhibited very high aqueous solubility [16].

In Fig. 2a, panels 1-2 present digital images of the MdGO suspension ( $1 \mathrm{mg} \mathrm{mL}^{-1}$ ) after mild sonication of 0 and $6 \mathrm{~h}$ standing, respectively. No obvious precipitate was observed, testifying the high solubility of MdGO. In addition, high stability in aqueous solution was observed for the MdGO suspension with the addition of $\mathrm{Co}^{2+}$ ions reaching $1 \mathrm{mmol} \mathrm{L}^{-1}$, which can be hardly attained by applying $\mathrm{HGO}$, as displayed in Fig. $2 \mathrm{a}(3,4)$. Combining the high functionalities and ratio of $\mathrm{C}=\mathrm{O} / \mathrm{C}-\mathrm{O}$, the dark black color and good aqueous solubility, even with the disturbance of transition metal ions, we may safely conclude that the main functional groups are located at the edge region [16]. Electron microscopy (EM) characterization of MdGO was then performed to examine its morphology. As observed in Figs $2 b$ and c, MdGO exhibits a typical thin layer morphology with a smooth basal plane. Because of the reduced functionality content on the basal plane, MdGO exhibits a widely spanned interlayer space from 0.78 to $0.34 \mathrm{~nm}$, calculated using Bragg's equation (5) based on the XRD patterns in Fig. 2d. In contrast, for the HGO, the XRD results revealed a typical (002) peak at $11^{\circ}$, which was indexed to the $0.81 \mathrm{~nm}$ interlayer distance, revealing many more functional groups on the basal planes than on those of MdGO. The layer thickness was measured using atomic force microscopy (AFM). As observed in Fig. 2e, three transection lines were made, resulting in a section depth of $\sim 1.87 \mathrm{~nm}$. By combining the interlayer space distance of 0.34 to 0.78 $\mathrm{nm}$, we can obtain the layer number lying in 2-5 layers.

$$
d=\frac{\lambda}{2 \sin \theta} .
$$

Before being used as a catalyst support, the MdGO was pretreated under hydrothermal conditions in the presence of $\mathrm{NH}_{3} \cdot \mathrm{NH}_{3}$ can tune the system $\mathrm{pH}$ to strong alkalinity and sufficiently doped MdGO with $\mathrm{N}$ [4]. As demonstrated in Figs $3 \mathrm{a}$ and $\mathrm{b}$, the $\mathrm{N}$-doped MdGO (N-rMdGO) remains in layered form but exhibits a certain degree of crumpling. The XPS C1s spectrum in Fig. 3c clearly reveals the effective defunctionalization of MdGO, especially the removal of carboxyl groups (attributed to the binding energy of $\sim 288.5$ $\mathrm{eV}$, marked by the arrow). In addition, the configuration of 

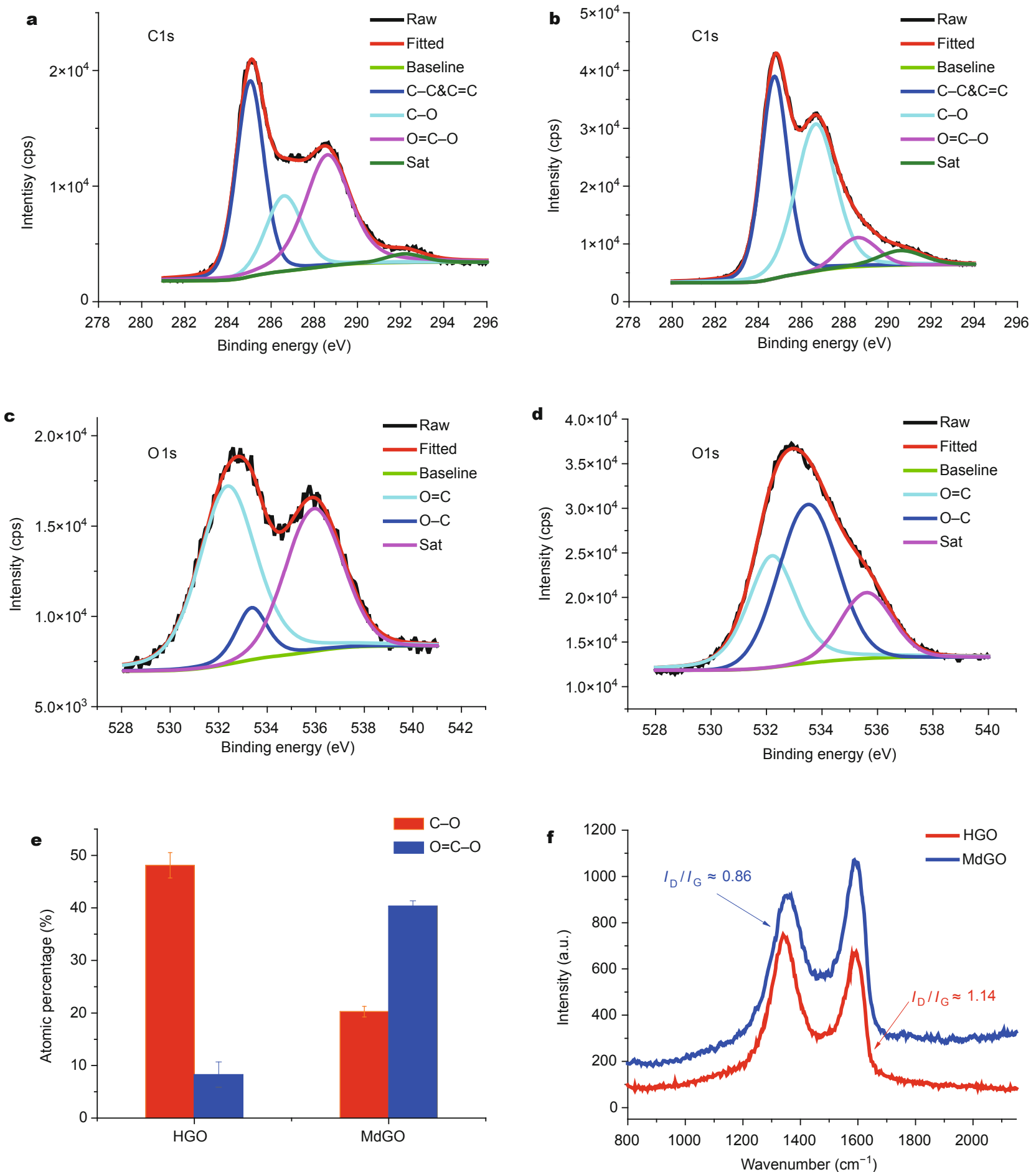

Figure 1 Bonding configurations and defect analysis of MdGO and HGO. XPS C1s spectra of (a) HGO and (b) MdGO. XPS O1s spectra of (c) HGO and (d) MdGO. (e) Percentage of $\mathrm{C}-\mathrm{O}$ (red) and $\mathrm{O}=\mathrm{C}-\mathrm{O}$ (blue) in overall C content of HGO and MdGO. (f) Raman spectra of HGO and MdGO. 

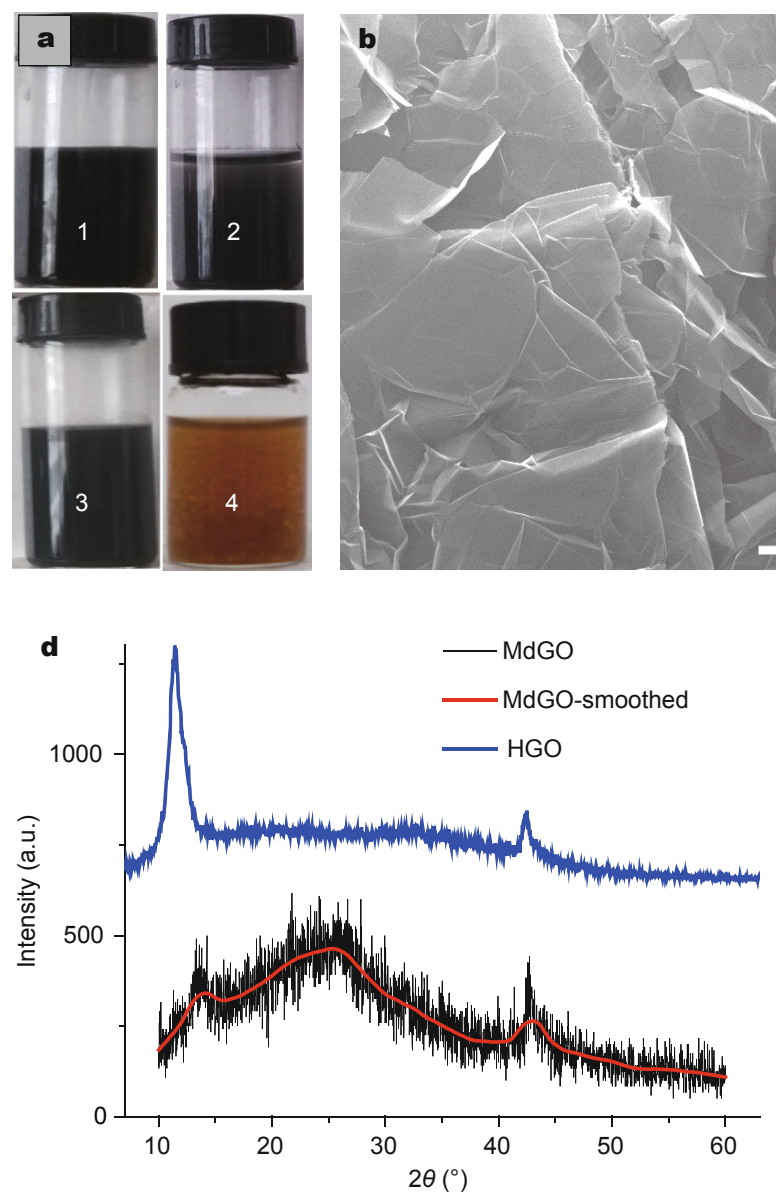
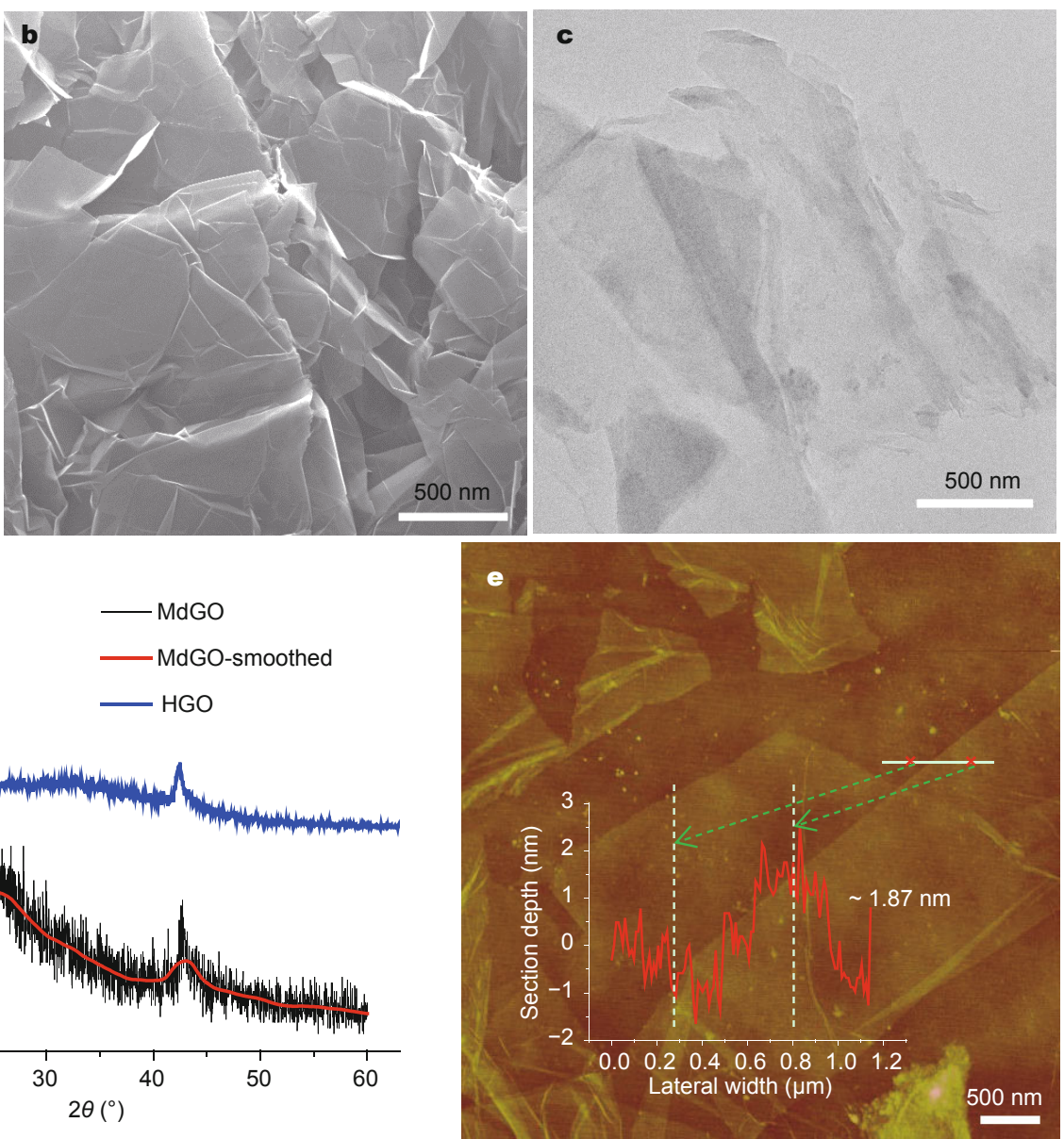

Figure 2 Characterizations of modified synthetic graphene oxide (MdGO) and Hummers' GO (HGO). (a) Stability of MdGO in aqueous solution under various conditions: (1) right after sonication; (2) $6 \mathrm{~h}$ after sonication; (3) upon adding $\mathrm{Co}^{2+}$ to reach $1 \mathrm{~mol} \mathrm{~L}^{-1} \mathrm{Co}^{2+}$ in $\mathrm{MdGO}^{2}$ suspension, where no precipitate was formed; (4) upon adding $\mathrm{Co}^{2+}$ to reach $1 \mathrm{~mol} \mathrm{~L}^{-1} \mathrm{Co}^{2+}$ in $\mathrm{HGO}$ suspension, in which floccule can be clearly seen at the bottom. (b) SEM and (c) TEM images of MdGO. (d) XRD patterns of MdGO and HGO. (e) AFM image of MdGO. The inset lines represent the three intersection line in (e) and the section depths were $\sim 1.87 \mathrm{~nm}$.

$\mathrm{N}$ mainly takes the pyrrolic form, as shown in Fig. 3d. Catalyst loading was performed using the dip coating method. The coating condition was set in $\mathrm{Co}^{2+} / \mathrm{DMSO}$ solution at $80^{\circ} \mathrm{C}$. Once the adsorption equilibrium was reached after a sufficient duration $(2 \mathrm{~h})$, the N-rMdGO or N-rHGO with $\mathrm{Co}^{2+}$ adsorption layer was separated from the $\mathrm{Co}^{2+}$ I DMSO solution using one-time centrifugation. The dried precursors were then annealed at $500^{\circ} \mathrm{C}$ for $2 \mathrm{~h}$ under inert conditions. Because of the reduced functionalities and the medium viscosity of DMSO, thin layer deposition of $\mathrm{Co}^{2+}$ and transformation into unstable particles can be achieved at low temperature. The formation of a single void inside the $\mathrm{CoO}$ nanocrystals (Fig. 4a) is generally attributed to the nanoscale Kirkendall effect, which has proven to be a versatile method for the generation of hollow inorganic nanocrystals [22]. The SEM images in Fig. 4 b reveal the uniform and sparsely packed $\mathrm{CoO}$ particles. Although the N-rHGO will adsorb dense Co species, nanoparticles will be readily formed on the thin HGO layer, as shown in Fig. 4c. In addition, after inert annealing, the N-rHGO composite exhibited much less solubility even in strong polar DMF because of the high attaching behavior between N-rHGO sheets. The phase of the Co species was revealed to be $\mathrm{CoO}$ (Fig. 4d). The CoO species, as indicated in Fig. 4e, takes up only $\sim 1.39$ at. $\%$ of the overall materials in CoO/N-rMdGO.

The effectiveness of $\mathrm{CoO} / \mathrm{N}-\mathrm{rMdGO}$ hybrids on the ORR was determined in $0.1 \mathrm{~mol} \mathrm{~L}^{-1} \mathrm{KOH}$ and compared with that of $\mathrm{CoO} / \mathrm{N}-\mathrm{rHGO}$ and the standard catalyst 20 wt.\% Pt/C. The difference in the electrochemical performance was first investigated using RDE measurements. As observed in Fig. 5a, the CV curves of CoO-decorated $\mathrm{N}$-rHGO and N-rMdGO present very large differences with respect to the current peaks and their maximum peak locations. CoO/N-rMdGO exhibites a much higher max- 
a
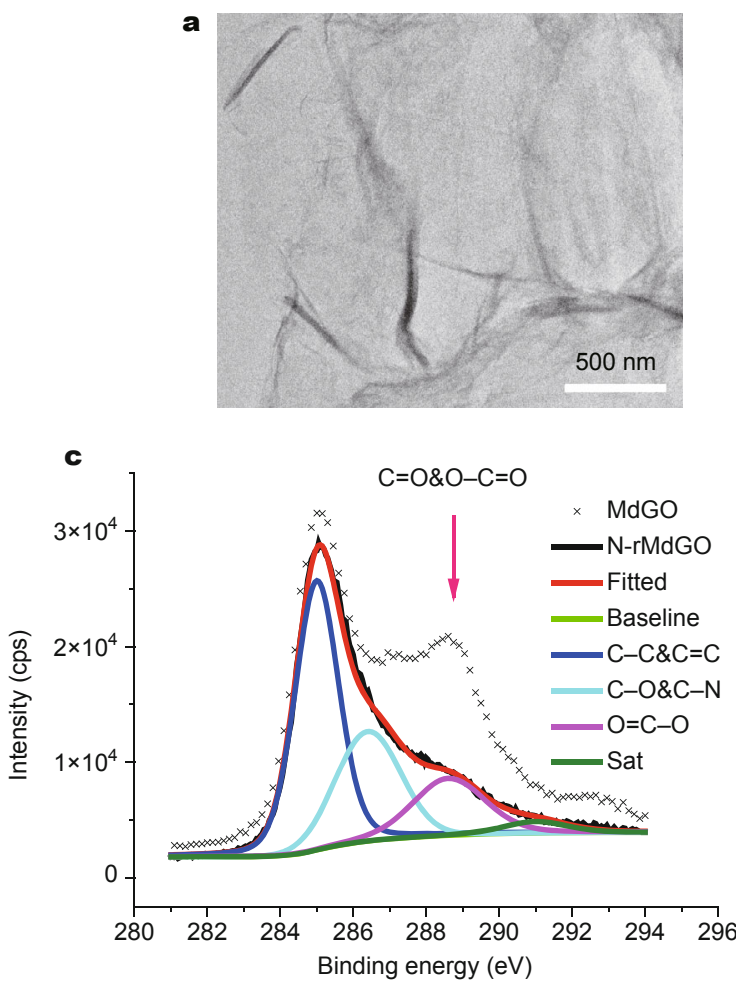
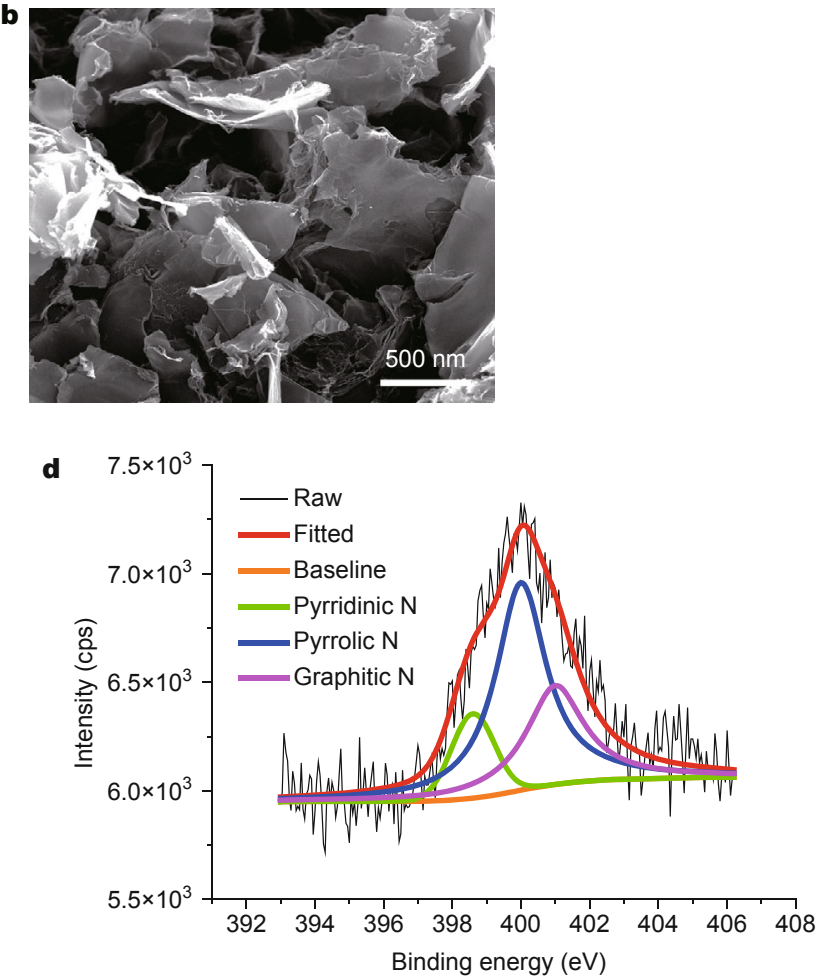

Figure 3 Characterizations of N-doped/reduced MdGO (N-rMdGO): (a) TEM image, (b) SEM image, (c) XPS C1s spectrum, and (d) XPS N1 spectrum.
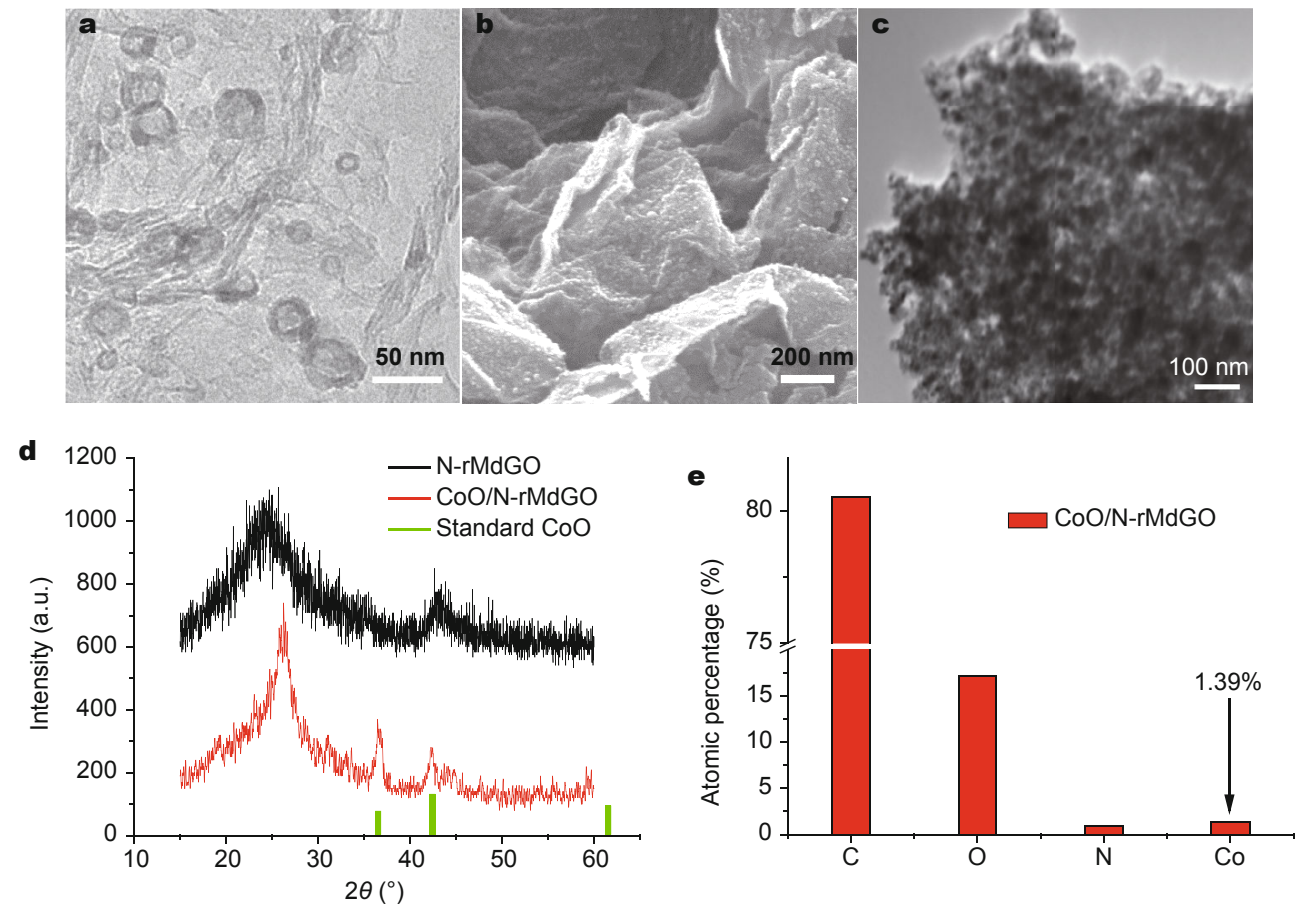

Figure 4 Characterizations of $\mathrm{CoO}$ anchored N-rMdGO (CoO/N-rMdGO) and N-rHGO (CoO/N-rHGO): (a) TEM and (b) SEM images of CoO/NrMdGO and (c) TEM image of CoO/N-rHGO. (d) XRD patterns of N-rMdGO and CoO/N-rMdGO. The standard CoO lines are provided for comparison. (e) XPS elemental results for CoO/N-rMdGO. 

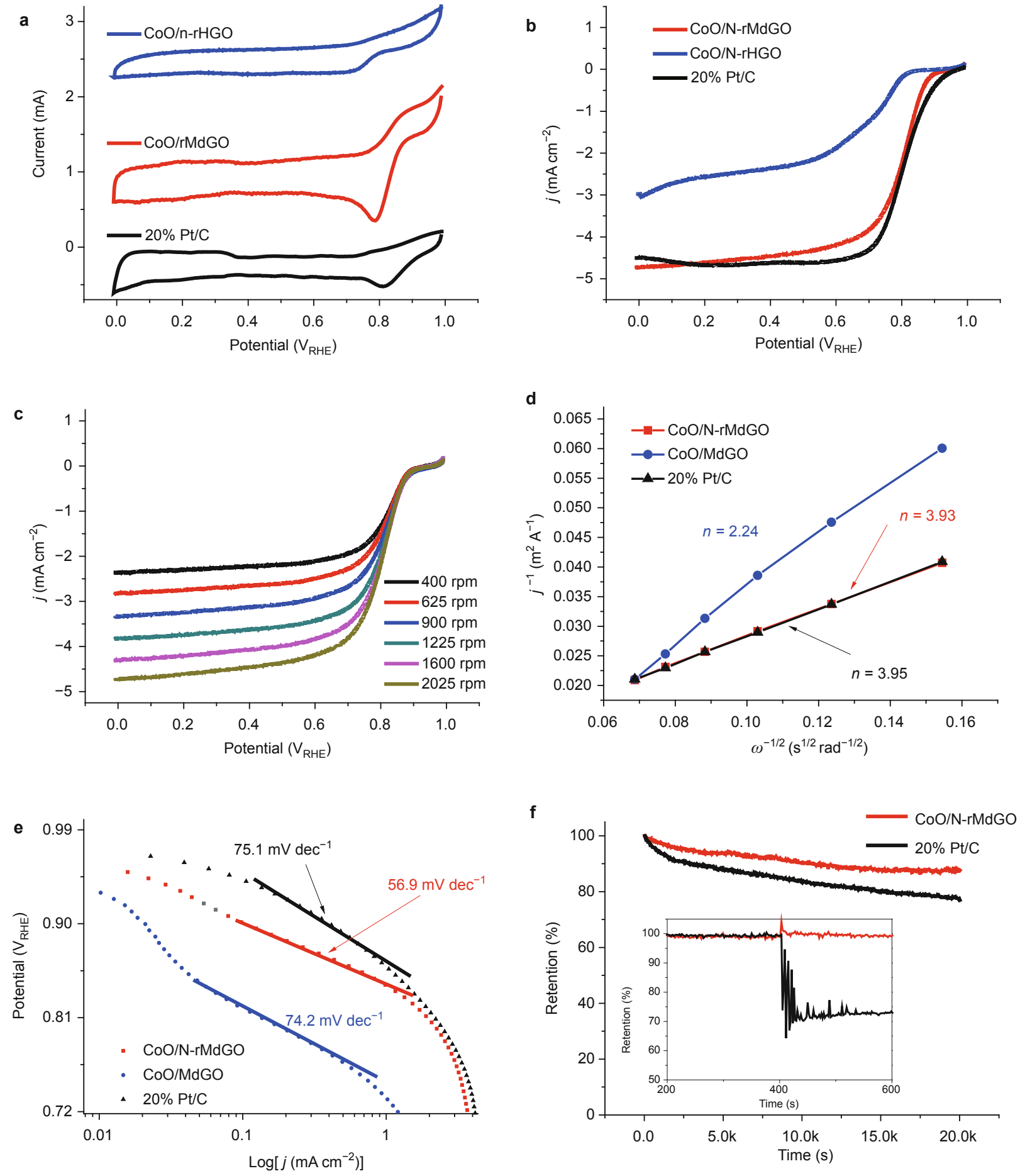

Figure 5 ORR performance of CoO/MdGO and CoO/N-rMdGO composites with reference to 20 wt.\% Pt/C. (a) CV and (b) LSV comparison; (c) LSV curves under various rotation rates; (d) K-L plots, with a potential of $0.6 \mathrm{~V}$ selected; (e) Tafel plots; (f) cycling stability. The inset in (f) shows the resistance to methanol. 
imum current and increased potential at maximum current. $\mathrm{CoO} / \mathrm{N}-\mathrm{rMdGO}$ even exhibits the nearest maximum current peak to that of $20 \% \mathrm{Pt} / \mathrm{C}$ at $\sim 0.8 \mathrm{~V}$. The variations can be more clearly seen in the linear sweep voltammetry spectra (LSV) in Fig. 5b. CoO/N-rMdGO achieves a half-wave potential of $0.82 \mathrm{~V}$ and a high limiting current density, which are both highly comparable to those of 20 wt.\% Pt/C. The RDE curves of CoO/N-rMdGO collected under various rotation rates are presented in Fig. $5 \mathrm{c}$ and are parallel to each other with an equal interval. Based on the paralleled LSV, $J^{-1} v s . \omega^{-1 / 2}$ was plotted to determine the efficiency of catalyzing the ORR. The standard four-electron transferring number can be calculated through LSV for $\mathrm{CoO} / \mathrm{N}-\mathrm{rMdGO}$ and $\mathrm{Pt} / \mathrm{C}$; however, insufficiency of the reaction efficiency was observed for CoO-deposited $\mathrm{N}$-rHGO, which may be due to the high resistance between the sheets. Remarkably, CoO/N-rMdGO possessed a lower Tafel slope than $\mathrm{Pt} / \mathrm{C}$, indicating faster ORR kinetics than those of $\mathrm{Pt} / \mathrm{C}$ in alkaline solution, as observed in Fig. 5e. Moreover, 20,000-s cycling only caused a $12.2 \%$ activity decrease relative to the $23.1 \%$ fading observed for $20 \mathrm{wt} . \%$ $\mathrm{Pt} / \mathrm{C}$, as shown in the inset of Fig. 5f. Methanol addition (inset of Fig. 5f) during cycling had a negligible effect on $\mathrm{CoO} / \mathrm{N}-\mathrm{rMdGO}$. However, a sharp drop was observed for 20 wt.\% Pt/C, which could only maintain roughly $70 \%$ of its initial activity with the addition of methanol.

\section{CONCLUSIONS}

The distribution of oxygen-containing functional groups on GO was manipulated using a modified synthetic strategy of long intercalation and short reaction duration. Most of the functionality of MdGO was verified in the form of carboxyl groups, which were two folds larger than the hydroxyl groups and located on the edge region. MdGO has a dark black color, high oxygen content, and very good aqueous solubility. GO with such a structure greatly facilitated the solution processibility while maintaining excellent conductivity. In addition, MdGO can serve as a very good catalyst support. The hydroxyl groups on the basal plane can anchor the catalyst, and the carboxyl groups can be easily removed after solution processes to form a highly active and conductive composite for catalysis. Demonstration of $\mathrm{CoO}$ catalyst loading was performed, and the composite $\mathrm{CoO} / \mathrm{N}-\mathrm{rMdGO}$ exhibited highly comparable performance to $20 \mathrm{wt} . \% \mathrm{Pt} / \mathrm{C}$, which indicates the high potential of MdGO as an excellent catalyst support.

\section{Received 26 May 2015; accepted 24 June 2015} published online 15 July 2015

1 Guo S, Zhang S, Wu L, Sun S. Co/CoO nanoparticles assembled on graphene for electrochemical reduction of oxygen. Angew Chem, 2012, 124: 11940-11943

2 He Q, Li Q, Khene S, et al. High-loading cobalt oxide coupled with nitrogen-doped graphene for oxygen reduction in anion-exchange-membrane alkaline fuel cells. J Phys Chem C, 2013, 117: 8697-8707

3 Jiang S, Zhu C, Dong S. Cobalt and nitrogen-cofunctionalized graphene as a durable non-precious metal catalyst with enhanced ORR activity. J Mater Chem A, 2013, 1: 3593-3599

4 Liang $\mathrm{Y}$, Li Y, Wang $\mathrm{H}$, et al. $\mathrm{Co}_{3} \mathrm{O}_{4}$ nanocrystals on graphene as a synergistic catalyst for oxygen reduction reaction. Nat Mater, 2011, 10: 780-786

5 Liang Y, Wang H, Zhou J, et al. Covalent hybrid of spinel manganese-cobalt oxide and graphene as advanced oxygen reduction electrocatalysts. J Am Chem Soc, 2012, 134: 3517-3523

6 Wang H, Liang Y, Li Y, Dai H. $\mathrm{Co}_{1-x} \mathrm{~S}$-graphene hybrid: a high-performance metal chalcogenide electrocatalyst for oxygen reduction. Angew Chem Int Ed, 2011, 50: 10969-10972

7 Wang G, Yang J, Park J, et al. Facile synthesis and characterization of graphene nanosheets. J Phys Chem C, 2008, 112: 8192-8195

8 Zhou X, Qiao J, Yang L, Zhang J. A review of graphene-based nanostructural materials for both catalyst supports and metal-free catalysts in pem fuel cell oxygen reduction reactions. Adv Energ Mater, 2014, 4: 1289-1295

9 Luo J, Kim J, Huang J. Material processing of chemically modified graphene: some challenges and solutions. Acc Chem Res, 2013, 46: 2225-2234

10 Wang H, Dai H. Strongly coupled inorganic-nano-carbon hybrid materials for energy storage. Chem Soc Rev, 2013, 42: 3088-3113

11 Marcano DC, Kosynkin DV, Berlin JM, et al. Improved synthesis of graphene oxide. ACS Nano, 2010, 4: 4806-4814

12 Luo D, Zhang G, Liu J, Sun X. Evaluation criteria for reduced graphene oxide. J Phys Chem C, 2012, 115: 11327-11335.

13 Pei S, Cheng HM. The reduction of graphene oxide. Carbon, 2012, 50: 3210-3228.

14 Chua CK, Pumera M. Chemical reduction of graphene oxide: a synthetic chemistry viewpoint. Chem Soc Rev, 2014, 43: 291-312

15 Gao W, Alemany LB, Ci L, Ajayan PM. New insights into the structure and reduction of graphite oxide. Nat Chem, 2009, 1: 403-408

16 Jeon IY, Shin YR, Sohn GJ, et al. Edge-carboxylated graphene nanosheets via ball milling. Proc Natl Acad Sci, 2012, 109: 55885593

17 Huang NM, Lim HN, Chia CH, Yarmo MA, Muhamad MR. Simple room-temperature preparation of high-yield large-area graphene oxide. Int J Nanomed, 2010, 6: 3443-3448

18 Sun X, Zhuang L, Welsher K, et al. Nano-graphene oxide for cellular imaging and drug delivery. Nano Res, 2008, 1: 203-212.

19 Dimiev AM, Tour JM. Mechanism of graphene oxide formation. ACS Nano, 2014, 8: 3060-3068

20 Silvestre-Albero J. Ultrahigh $\mathrm{CO}_{2}$ adsorption capacity on carbon molecular sieves at room temperature. Chem Commun, 2011, 47: 6840-6842

21 Xia Y, Mokaya R, Walker GS, Zhu Y. Superior $\mathrm{CO}_{2}$ adsorption capacity on $\mathrm{N}$-doped, high-surface-area, microporous carbons templated from zeolite. Adv Energ Mater, 2011, 1: 678-683

22 Yang Z, Lisiecki I, Walls M, Pileni MP. Nanocrystallinity and the ordering of nanoparticles in two-dimensional superlattices: controlled formation of either core/shell $(\mathrm{Co} / \mathrm{CoO})$ or hollow $\mathrm{CoO}$ nanocrystals. ACS Nano, 2013, 7: 1342-1350

Acknowledgements This work was supported by the National Basic Research Program of China (2011CBA00503 and 2011CB932403), the 
Program for Changjiang Scholars and Innovative Research Team in the University (IRT1205), and the Fundamental Research Funds for the Central Universities (YS1406).

Author contributions Zhang G designed the experiments, and Zhang G, $\mathrm{Xu} \mathrm{Y}$, and Wang $\mathrm{L}$ performed the experiments and the characterization.
Wang J helped with the sample characterization. Zhang G wrote the paper with support from Sun X. All the authors contributed to the general discussion.

Conflict of interest The authors declare that they have no conflict of interest.

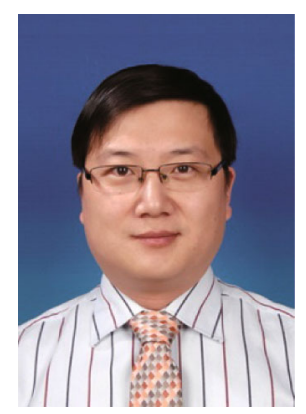

Xiaoming Sun was born in Pingdu, Shandong Province, China. He received his BSc and $\mathrm{PhD}$ degrees from the Department of Chemistry, Tsinghua University in 2000 and 2005, respectively. After post-doctoral work in Prof. Hongjie Dai's group at Stanford University, he joined the faculty of Beijing University of Chemical Technology at 2008. His research interests focus on controlled synthesis, the separation and assembly of functional inorganic nanomaterials and carbon materials, and the structure and property regulation of metal-oxide arrays.

中文摘要 本文采用液相化学法对氧化石墨烯氧化官能团的分布进行控制合成, 使较多含氧官能团在氧化石墨烯的边缘区域. X射线 光电子能谱显示其具有高的含氧官能度, 且 $\mathrm{C}=\mathrm{O} / \mathrm{C}-\mathrm{O}$ 比值在 2 左右, 远高于原有Hummers方法制备的氧化石墨烯的 0.17 . 其深黑色的外 观与其他文献对比, 可以证明其主要的含氧官能团为羧基且羰基主要存在于边缘区域. 具有该结构的氧化石墨烯可以经过简单还原后, 极大地恢复其高导电性, 层面上存有的少量官能团可以用来有效地抓住催化剂颗粒, 使得由这种氧化石墨烯所制得的 $\mathrm{CoO}$ 复合物具有 非常优秀的催化氧气还原反应性能. 